\title{
Optimization through dense sets
}

\author{
C. R. Jayanarayanan \\ T. S. S. R. K. Rao
}

\begin{abstract}
In this paper, we study two optimization problems where solutions on a dense set yield global solution. We study these problems for spaces of Bochner integrable functions and for spaces of continuous functions. The first one deals with expressing the length of a vector as a sum of the distance to a best approximation and minimal best approximation and the second one relates to approximating a subsequence of a minimizing sequence with a sequence of proximinal vectors.
\end{abstract}

\section{Introduction}

Let $X$ be a real Banach space and let $A \subseteq X$ be an absolutely convex and closed set. Let $A^{o}=\left\{f \in X^{*}:|f| \leq 1\right.$ on $\left.A\right\}$. Let $A^{o o}$ be the double polar in $X^{* *}$. An important problem in optimization theory is to study the proximinality properties of $A$ vis a vis those of $A^{o o}$. Clearly because bounded weak*-closed sets in $X^{* *}$ are weak*-compact, $A^{o o}$ is better suited for the study of optimization problem (in particular it is a proximinal set). We assume that $X$ is canonically embedded in $X^{* *}$ via the map $x \rightarrow \widehat{x}$, where $\widehat{x}$ is the evaluation map on $X^{*}$. It is well known that $X$ under this embedding is a weak*-dense subspace of $X^{* *}$. For notational convenience, we ignore the embedding and denote $\widehat{x}$ by $x$ itself. In this paper, we study a variation on the classical theme by studying optimization problems for $A^{o o}$ where the constraints are imposed only on vectors in $X$. We mainly deal with the situation when $A=Y$ is a closed subspace so that $A^{o o}=Y^{\perp \perp}$. Throughout the paper, we shall assume all subspaces are closed.

Received by the editors in March 2016 - In revised form in June 2016.

Communicated by G. Godefroy.

2010 Mathematics Subject Classification : Primary 41A65, secondary 46E40, 46B20, 41A50.

Key words and phrases : Proximinality, strong proximinality, space of Bochner integrable functions, space of continuous functions. 
We note that $Y^{\perp \perp}$ is isometrically identified with $Y^{* *}$. It is well known that optimization is quite a delicate operation under perturbation and even existence of best approximation at a dense set of points need not imply proximinality. In this paper, we study two stronger forms of proximinality, that allow us to make the passage from a dense set to the whole space. For $x \in X$, let $P_{A}(x)$ denote the set of best approximations to $x$ in $A$ (possibly empty set). Elements of $P_{A}(x)$ are called proximinal vectors. When $A$ is a proximinal set in $X$, we recall from [5] that $A$ has property $(*)$ at $x$, if $\|x\|=d(x, A)+d\left(0, P_{A}(x)\right)$. In Section 2 , we show that for any complete probability measure space $(\Omega, \mathcal{A}, \mu)$ and for any subspace $Y \subseteq X, Y$ has property $(*)$ in $X$ implies that the space of Bochner integrable functions $L_{1}(\mu, Y)$ has property $(*)$ at all simple functions in $L_{1}(\mu, X)$. If, in addition, $Y$ is separable, then $L_{1}(\mu, Y)$ has property $(*)$ in $L_{1}(\mu, X)$. We also show that for a closed subspace $Y \subset X$, if $Y^{\perp \perp}$ has property $(*)$ for all $x \in X$, then it has property $(*)$ at all points of $X^{* *}$.

Let $B_{X}$ denote the closed unit ball of $X$ and $B(x, r)$ denote the closed ball with center at $x \in X$ and radius $r>0$. Ignoring the canonical embedding of $X$ in $X^{* *}$, we continue to denote by $B(x, r)$ the larger closed ball in $X^{* *}$ with center at $x$. The distinction will be clear from the context.

We next consider the following weaker form of property $(*)$, which amounts to approximating a subsequence of a minimizing sequence with a sequence of proximinal vectors.

Definition 1.1 ([4]). We recall that a proximinal subspace $Y$ of a Banach space $X$ is said to be strongly proximinal at $x \in X$ if for every $\varepsilon>0$, there exists a $\delta>0$ such that $P_{Y}(x, \delta) \subseteq P_{Y}(x)+\varepsilon B_{X}$, where $P_{Y}(x, \delta)=\{y \in Y:\|x-y\|<d(x, Y)+\delta\}$. If $Y$ is strongly proximinal at all points of $X$, we say that $Y$ is strongly proximinal in $X$.

Fact 1.2 ([4]). Let $Y$ be a proximinal subspace of a Banach space $X, x \in X$ and $\varepsilon>0$. Now let $Q_{Y}(x)=x-P_{Y}(x)$ and $Q_{Y}(x, \varepsilon)=x-P_{Y}(x, \varepsilon)$. Moreover, for an $x \in X$ with $\|x\|=1=d(x, Y)$, we have

$$
\begin{gathered}
Q_{Y}(x)=\left\{z \in X:\|z\|=1 \text { and } f(z)=f(x) \text { for all } f \in Y^{\perp}\right\}, \\
Q_{Y}(x, \varepsilon)=\left\{z \in X:\|z\| \leq 1+\varepsilon \text { and } f(z)=f(x) \text { for all } f \in Y^{\perp}\right\} .
\end{gathered}
$$

Now it follows that $Y$ is strongly proximinal at $x$ if and only if $\lim _{\varepsilon \rightarrow 0}$ sup $\left\{d\left(z, Q_{Y}(x)\right): z \in Q_{Y}(x, \varepsilon)\right\}=0$.

It is easy to see that property $(*)$ implies strong proximinality. It is known that this property is easier to handle and better understood when $Y$ is a subspace of finite co-dimension (see [4]). For a compact Hausdorff space $K$, we shall denote by $C(K)$ the space of all continuous functions on $K$ equipped with the supremum norm and it is known that its dual $C(K)^{*}$ is the space of all regular Borel measures on $K$. In Section 3, we show that for a finite co-dimensional proximinal subspace $Y$ of $C(K)$, if $Y^{\perp \perp}$ is strongly proximinal at all points of $C(K)$, then $Y^{\perp} \subseteq\left\{\mu \in C(K)^{*}: \mu\right.$ is finitely supported $\}$. In a general set up, we also show that if $W \subset X^{* *}$ is a weak*-closed subspace of finite co-dimension in $X^{* *}$, then under some additional conditions, strong proximinality of $W$ at points of $X$, implies strong proximinality of $W$ in $X^{* *}$. 


\section{Invariance of property $(*)$}

In this section, we start by giving an example of a Banach space and a subspace for which the existence of a best approximation for all points of a dense subset need not imply proximinality of the subspace. Let $(\Omega, \mathcal{A}, \mu)$ be a finite positive measure space and let $L_{1}(\mu, X)$ denote the space of $X$-valued Bochner integrable functions on $\Omega$.

Example 2.1. If $Y$ is a proximinal subspace of a Banach space $X$, then every simple function in $L_{1}(\mu, X)$ has a best approximation from $L_{1}(\mu, Y)$. Indeed, let $s=\sum_{i=1}^{n} x_{i} \chi_{A_{i}}$, where $x_{i} \in X(1 \leq i \leq n)$ and $\left\{A_{1}, \ldots, A_{n}\right\}$ is a finite family of mutually disjoint measurable sets. For $1 \leq i \leq n$, let $y_{i} \in P_{Y}\left(x_{i}\right)$. Then, by [9, Corollary 2.11], $s^{\prime}=\sum_{i=1}^{n} y_{i} \chi_{A_{i}}$ is a best approximation for $s$ from $L_{1}(\mu, Y)$. In [10, Example 3.1], Mendoza gave an example of a Banach space $X$ and a proximinal subspace $Y$ of $X$ such that $L_{1}([0,1], Y)$ is not proximinal in $L_{1}([0,1], X)$. Thus we have that $L_{1}([0,1], Y)$ is not proximinal in $L_{1}([0,1], X)$ even though the set $\left\{f \in L_{1}([0,1], X): P_{L_{1}([0,1], Y)}(f) \neq \varnothing\right\}$ is dense in $L_{1}([0,1], X)$.

Let $(\Omega, \mathcal{A}, \mu)$ be a finite positive measure space and let $Y$ be a subspace of $X$. In what follows, we will be using the integral formula, for $f \in L_{1}(\mu, X)$, $d\left(f, L_{1}(\mu, Y)\right)=\int_{\Omega} d(f(t), Y) d \mu$, and $g \in P_{L_{1}(\mu, Y)}(f)$ if and only if $g(t) \in P_{Y}(f(t))$ for almost all $t \in \Omega$ (see [9, Lemma 2.10, Corollary 2.11]).

We next show that for a subspace $Y$ of a Banach space $X$ with property $(*)$ in $X, L_{1}(\mu, Y)$ has property $(*)$ at all points of a dense subsets of $L_{1}(\mu, X)$.

Theorem 2.2. Let $(\Omega, \mathcal{A}, \mu)$ be a finite positive measure space. Let $Y$ be a subspace of a Banach space $X$ such that $Y$ has property $(*)$ in $X$. Then $L_{1}(\mu, Y)$ has property $(*)$ at all simple functions in $L_{1}(\mu, X)$.

Proof. By hypothesis as $Y$ is proximinal in $X$, it follows from Example 2.1 that every simple function in $L_{1}(\mu, X)$ has a best approximation from $L_{1}(\mu, Y)$. Let $s=\sum_{i=1}^{n} x_{i} \chi_{A_{i}}$, where $x_{i} \in X(1 \leq i \leq n)$ and $\left\{A_{1}, \ldots, A_{n}\right\}$ is a finite family of mutually disjoint measurable sets. For $1 \leq i \leq n$, let $y_{i} \in P_{Y}\left(x_{i}\right)$.

Claim: $d\left(0, P_{L_{1}(\mu, Y)}(s)\right)=\sum_{i} d\left(0, P_{Y}\left(x_{i}\right)\right) \mu\left(A_{i}\right)$.

For, let $g \in P_{L_{1}(\mu, Y)}(s)$. Then, by [9, Corollary 2.11], $g(t) \in P_{Y}(s(t))$ for almost all $t \in \Omega$. Thus $\|g\|=\int_{\Omega}\|g(t)\| d \mu \geq \int_{\Omega} d\left(0, P_{Y}(f(t)) d \mu=\sum_{i} d\left(0, P_{Y}\left(x_{i}\right)\right) \mu\left(A_{i}\right)\right.$. Hence $d\left(0, P_{L_{1}(\mu, Y)}(f)\right) \geq \sum_{i} d\left(0, P_{Y}\left(x_{i}\right)\right) \mu\left(A_{i}\right)$.

For the reverse inequality suppose $\varepsilon>0$. Then there exists a $y_{i} \in P_{Y_{i}}\left(x_{i}\right)$ such that $\left\|y_{i}\right\|<d\left(0, P_{Y}\left(x_{i}\right)\right)+\frac{\varepsilon}{n \mu(\Omega)}$. Thus, by [9, Corollary 2.11], $s^{\prime}=\sum_{i=1}^{n} y_{i} \chi_{A_{i}}$ is a best approximation for $s$ from $L_{1}(\mu, Y)$. Hence

$$
\begin{aligned}
\left\|s^{\prime}\right\|=\sum_{i}\left\|y_{i}\right\| \mu\left(A_{i}\right) & \leq \sum_{i}\left(d\left(0, P_{Y}\left(x_{i}\right)\right)+\frac{\varepsilon}{n \mu(\Omega)}\right) \mu\left(A_{i}\right) \\
& \leq \sum_{i} d\left(0, P_{Y}\left(x_{i}\right)\right) \mu\left(A_{i}\right)+\varepsilon .
\end{aligned}
$$


Hence the claim follows. Now

$$
\begin{aligned}
\|s\|=\sum_{i}\left\|x_{i}\right\| \mu\left(A_{i}\right) & =\sum_{i} d\left(x_{i}, Y_{i}\right) \mu\left(A_{i}\right)+\sum_{i} d\left(0, P_{Y_{i}}\left(x_{i}\right)\right) \mu\left(A_{i}\right) \\
& =d\left(s, L_{1}(\mu, Y)\right)+d\left(0, P_{L_{1}(\mu, Y)}(s)\right) .
\end{aligned}
$$

The last equality above is a consequence of the integral formula for distance function applied to the case of a simple function.

As an application of von Neumann's selection theorem (see [12] *Corollary 5.5.8), we next show that if $Y$ is a separable subspace of a Banach space $X$ having property $(*)$ in $X$ and the positive measure space is complete, then $L_{1}(\mu, Y)$ has property $(*)$ in $L_{1}(\mu, X)$. We now recall that under the same assumptions on $Y$, by [10, Theorem 3.4], $L_{1}(\mu, Y)$ is proximinal in $L_{1}(\mu, X)$.

Theorem 2.3. Let $(\Omega, \mathcal{A}, \mu)$ be a complete probability measure space. Let $Y$ be a separable subspace of a Banach space X such that $Y$ has property $(*)$ in $X$. Then $L_{1}(\mu, Y)$ has property $(*)$ in $L_{1}(\mu, X)$.

Proof. Let $f \in L_{1}(\mu, X)$. As $f$ is an almost everywhere separably valued function, by discarding a null set we may assume that $f$ is everywhere defined. Since, by hypothesis, $d\left(0, P_{Y}(f(t))=\|f(t)\|-d(f(t), Y)\right.$ for almost all $t \in \Omega$, the function $t \rightarrow d\left(0, P_{Y}(f(t))\right.$ is integrable.

We now prove the integral version of the claim stated in Theorem 2.2.

Claim: $d\left(0, P_{L_{1}(\mu, Y)}(f)\right)=\int_{\Omega} d\left(0, P_{Y}(f(t)) d \mu\right.$.

For, let $g \in P_{L_{1}(\mu, Y)}(f)$. Then, by [9, Corollary 2.11], $g(t) \in P_{Y}(f(t))$ for almost all $t \in \Omega$. Thus $\|g\|=\int_{\Omega}\|g(t)\| d \mu \geq \int_{\Omega} d\left(0, P_{Y}(f(t)) d \mu\right.$. Hence $d\left(0, P_{L_{1}(\mu, Y)}(f)\right) \geq$ $\int_{\Omega} d\left(0, P_{Y}(f(t)) d \mu\right.$.

For the reverse inequality suppose $\varepsilon>0$. Now define a set valued map $H: \Omega \rightarrow 2^{\Upsilon}$ by

$$
H(t)=\left\{y \in P_{Y}(f(t)):\|y\| \leq d\left(0, P_{Y}(f(t))\right)+\varepsilon\right\} \quad \text { for } t \in \Omega .
$$

Since $Y$ is proximinal in $X, H(t) \neq \varnothing$ for all $t \in \Omega$. We now use von Neumann selection theorem (see [12, Corollary 5.5.8]) to obtain a measurable selection for the set valued map $H$. Since $Y$ is a separable Banach space, to use von Neumann selection theorem, it is enough to show that the graph of $H,\{(t, y) \in \Omega \times Y: y \in$ $H(t)\}=\left\{(t, y) \in \Omega \times Y:\|f(t)-y\|=d(f(t), Y)\right.$ and $\left.\|y\| \leq d\left(0, P_{Y}(f(t))\right)+\varepsilon\right\}$ is a measurable set in the product space. As all the functions involved in the description of the graph of $H$ are measurable, it follows that the graph of $H$ is a measurable set in the product space. Then there exists a measurable function $h: \Omega \rightarrow Y$ such that $h(t) \in H(t)$ for all $t \in \Omega$. Since $h$ is separably valued, it is strongly measurable. Now, since $h(t) \in P_{Y}(f(t))$ for all $t \in \Omega$, by [8, Lemma 1], we get $h \in P_{L_{1}(\mu, Y)}(f)$. Then the claim follows from the fact that $\|h\| \leq \int_{\Omega}\|h(t)\| d \mu \leq \int_{\Omega} d\left(0, P_{Y}(f(t))\right) d \mu+\varepsilon$. 
Now

$$
\begin{aligned}
\|f\| & =\int_{\Omega}\|f(t)\| d \mu \\
& =\int_{\Omega} d(f(t), Y) d \mu+\int_{\Omega} d\left(0, P_{Y}(f(t)) d \mu\right. \\
& =d\left(f, L_{1}(\mu, Y)\right)+d\left(0, P_{L_{1}(\mu, Y)}(f)\right) .
\end{aligned}
$$

Remark 2.4. Since only countably many functions are involved in the proofs of the above theorems and as Bochner integrable functions have $\sigma$-finite support, Theorem 2.2 and Theorem 2.3 are valid for any complete measure space.

We next prove that property $(*)$ is invariant under certain weak*-dense subsets. Our exposition requires some notions from [5]. We start by recalling the following equivalent form of property $(*)$ from [13].

Definition 2.5. A subspace $Y$ of a Banach space $X$ is said to have the $1 \frac{1}{2}$-ball property if the conditions $x \in X, y \in Y, r, s>0$ with $\|x-y\|<r+s$ and $B(x, r) \cap Y \neq \varnothing$ imply that $B(x, r) \cap B(y, s) \cap Y \neq \varnothing$.

Theorem 3 of [13] shows that a subspace $Y$ of $X$ has the $1 \frac{1}{2}$-ball property in $X$ if and only if $Y^{\perp \perp}$ has the $1 \frac{1}{2}$-ball property in $X^{* *}$. In the next set of results we prove a strong version of this by showing that, for a subspace $Y$ of $X$, if the conditions in the definition of the $1 \frac{1}{2}$-ball property for $Y^{\perp \perp}$ in $X^{* *}$ are satisfied when one ball has center from $X$ and the other one has center from $Y$, then $Y^{\perp \perp}$ still has the $1 \frac{1}{2}$-ball property in $X^{* *}$.

Lemma 2.6. Let $X$ be a Banach space and $Z$ be a proximinal subspace of $X^{* *}$. Then the following are equivalent.

(i) For every $x \in X, r>0, s>0$ with $\|x\|<r+s$ and $B(x, r) \cap Z \neq \varnothing$;

$$
B(x, r) \cap B(0, s) \cap Z \neq \varnothing .
$$

(ii) For every $x \in X,\|x\|=d(x, Z)+d\left(0, P_{Z}(x)\right)$.

(iii) For every $x \in X, z \in Z, r>0, s>0$ with $\|x-z\|<r+s$ and $B(x, r) \cap Z \neq \varnothing$;

$$
B(x, r) \cap B(z, s) \cap Z \neq \varnothing .
$$

Proof. (i) $\Longrightarrow$ (ii) Suppose (i) holds. Let $x \in X$. Now let $\varepsilon>0$. Then there exists a $\Lambda_{\varepsilon} \in P_{Z}(x)$ such that $\left\|\Lambda_{\varepsilon}\right\|<d\left(0, P_{Z}(x)\right)+\varepsilon$. Then

$$
\begin{aligned}
\|x\|-d(x, Z) & =\|x\|-\left\|x-\Lambda_{\varepsilon}\right\| \\
& \leq\left\|\Lambda_{\varepsilon}\right\| \leq d\left(0, P_{Z}(x)\right)+\varepsilon .
\end{aligned}
$$

Thus $\|x\| \leq d(x, Z)+d\left(0, P_{Z}(x)\right)$.

Now suppose $\|x\|<d(x, Z)+d\left(0, P_{Z}(x)\right)$. Let $\eta>0$ be such that $\|x\|-d(x, Z)+\eta<d\left(0, P_{Z}(x)\right)$. Now set $r=d(x, Z)$ and $s=\|x\|-d(x, Z)+\eta$. Then $\|x\|<r+s$ and $B(x, r) \cap Z \neq \varnothing$. Hence, by (i), there exists a $\Lambda_{0} \in B(x, r) \cap$ 
$B(0, s) \cap Z$. Since $\left\|x-\Lambda_{0}\right\| \leq r=d(x, Z), \Lambda_{0} \in P_{Z}(x)$. Then $\left\|\Lambda_{0}\right\| \leq s<$ $d\left(0, P_{Z}(x)\right)$, which is a contradiction.

(ii) $\Longrightarrow$ (iii) Suppose (ii) holds. Let $x \in X, z \in Z, r, s>0$ be such that $\|x-z\|<r+s$ and $B(x, r) \cap Z \neq \varnothing$. Then $d(x, Z) \leq r$. Without loss of generality, we assume $\|x-z\|>r$. Let $\varepsilon=r-d(x, Z)$ and let $C=\{\Lambda \in Z:\|x-\Lambda\| \leq$ $d(x, Z)+\varepsilon\}$. Then we claim that $d(z, C) \leq\|x-z\|-r$. If not, then there exists an $\eta>0$ such that $\|x-z\|-r+\eta<d(z, C)$. Let $\Lambda_{0} \in P_{Z}(x)$ be such that $\left\|z-\Lambda_{0}\right\|<d\left(z, P_{Z}(x)\right)+\eta$. Now let $\Lambda^{\prime}=\Lambda_{0}+\frac{\varepsilon\left(z-\Lambda_{0}\right)}{\left\|z-\Lambda_{0}\right\|}$. Then $\Lambda^{\prime} \in C$. Thus

$$
\begin{aligned}
d(z, C) & \leq\left\|z-\Lambda^{\prime}\right\| \\
& =\left\|z-\Lambda_{0}\right\|-\varepsilon \\
& \leq d\left(z, P_{Z}(x)\right)+\eta-r+d(x, Z) \\
& \leq d\left(0, P_{Z}(x-z)\right)+\eta-r+d(x-z, Z) \\
& =\|x-z\|-r+\eta,
\end{aligned}
$$

which is a contradiction to the assumption that $\|x-z\|-r+\eta<d(z, C)$. Therefore $d(z, C) \leq\|x-z\|-r<s$. Let $\widetilde{\Lambda} \in C$ be such that $\|z-\widetilde{\Lambda}\| \leq s$. Then $\|x-\widetilde{\Lambda}\| \leq d(x, Z)+\varepsilon=r$ and hence (iii) follows.

We thank the referee for suggesting this formulation of Lemma 2.6, which improves on an earlier version of ours. In what follows we will be using Lemma 2.6 for the proximinal subspace $Z=Y^{\perp \perp}$.

Theorem 2.7. Let $Y$ be a subspace of a Banach space $X$ such that for all $x \in X$, $\|x\|=d\left(x, Y^{\perp \perp}\right)+d\left(0, P_{Y^{\perp \perp}}(x)\right)$. Then for all $x^{* *} \in X^{* *},\left\|x^{* *}\right\|=d\left(x^{* *}, Y^{\perp \perp}\right)+$ $d\left(0, P_{Y \perp \perp}(x)\right)$.

Proof. We first show that $Y$ has the $1 \frac{1}{2}$-ball property in $X$. Let $x \in X, y \in Y$ and $r, s>0$ be such that $\|x-y\| \leq r+s$ and $B(x, r) \cap Y \neq \varnothing$. Also let $\varepsilon>0$. Since $Y^{\perp \perp}$ is a weak*-closed subspace of $X^{* *}$, it is proximinal and so by Lemma 2.6, there exists an element $\Lambda \in Y^{\perp \perp}$ such that $\Lambda \in B(x, r) \cap B(y, s) \cap Y^{\perp \perp}$. Let $E=\operatorname{span}\{x, y, \Lambda\}$ and $m=\max \{r, s\}$. Then, by an extended version of principle of local reflexivity (see [1, Theorem 3.2]), there exists an operator $T_{\epsilon}: E \rightarrow X$ such that

(1) $T_{\epsilon}(z)=z$ if $z \in E \cap X$,

(2) $T_{\epsilon}\left(E \cap Y^{\perp \perp}\right) \subseteq Y$,

(3) $\left\|T_{\epsilon}\right\| \leq 1+\frac{\epsilon}{r}$.

Now take $z=T_{\mathcal{E}}(\Lambda)$. Then $z \in B(x, r+\varepsilon) \cap B(y, s+\varepsilon) \cap Y$. Thus, by [13, Theorem 3], $Y$ has the $1 \frac{1}{2}$-ball property in $X$ and hence, by the same theorem, $Y^{\perp \perp}$ has the $1 \frac{1}{2}$-ball property in $X^{* *}$. Then, by [5, Corollary 3(i)], the theorem follows. 


\section{Strong proximinality}

In [3], A. L. Garkavi obtained the following characterization for finite co-dimensional proximinal subspaces of $C(K)$. See also [11, Page 302].

Theorem 3.1 ([3]). Let $K$ be a compact Hausdorff space and let $Y$ be a finite co-dimensional subspace of $C(K)$. Then $Y$ is proximinal in $C(K)$ if and only if the annihilator $Y^{\perp}$ satisfies the following three conditions:

(a) $\operatorname{supp}\left(\mu^{+}\right) \bigcap \operatorname{supp}\left(\mu^{-}\right)=\varnothing$ for each $\mu \in Y^{\perp} \backslash\{0\}$,

(b) $\mu$ is absolutely continuous with respect to $v$ on $\operatorname{supp}(v)$ for every pair $\mu, v \in Y^{\perp} \backslash\{0\}$,

(c) $\operatorname{supp}(v) \backslash \operatorname{supp}(\mu)$ is closed for each pair $\mu, v \in Y^{\perp} \backslash\{0\}$.

We now recall the following characterization of finite co-dimensional strongly proximinal subspace of $C(K)$.

Theorem 3.2 ([2]). Let $K$ be a compact Hausdorff space and let $Y$ be a finite co-dimensional subspace of $C(K)$. Then $Y$ is strongly proximinal in $C(K)$ if and only if $Y^{\perp} \subseteq\left\{\mu \in C(K)^{*}: \mu\right.$ is finitely supported $\}$.

Our next result shows that the above theorem is valid for weak ${ }^{*}$-closed subspaces of finite co-dimension in $C(K)^{* *}$ (which is again a space of continuous functions), under a weaker hypothesis. We first observe that for a Banach space $X$ and a finite co-dimensional weak*-closed subspace $W$ of $X^{* *}$, its annihilator $W^{\perp}$ in $X^{* * *}$ is actually contained in $X^{*}$. Thus a weak*-closed subspace of finite codimension in $X^{* *}$ is of the form $Y^{\perp \perp}$, where $Y$ is a finite co-dimensional subspace of $X$. We also recall from [7] that a finite co-dimensional proximinal subspace $Y$ of $X$ is strongly proximinal in $X$ if and only if $Y^{\perp \perp}$ is strongly proximinal in $X^{* *}$. In this section, we strengthen this result by considering strong proximinality of $Y^{\perp \perp}$ only at the points of $X$.

Theorem 3.3. Let $K$ be a compact Hausdorff space and let $Y$ be a finite co-dimensional proximinal subspace of $C(K)$. If $Y^{\perp \perp}$ is strongly proximinal at all points of $C(K)$, then $Y^{\perp} \subseteq\left\{\mu \in C(K)^{*}: \mu\right.$ is finitely supported $\}$.

We will prove the above theorem later as its proof requires several results that analyze the structure of subspaces of finite co-dimension which we will prove in a general setup.

Our exposition relies on a detailed study of strong proximinality for subspaces of finite co-dimension carried out in [4].

We now recall some notation from [6] which will play an important role in the rest of this section.

Let $X$ be a Banach space and $\left\{f_{1}, \ldots, f_{n}\right\}$ be a set of linearly independent functionals in $X^{*}$. Let $M_{1}=M_{1}^{*}=\left\|f_{1}\right\|, J_{X}\left(f_{1}\right)=\left\{x \in B_{X}: f_{1}(x)=\left\|f_{1}\right\|\right\}$ and $J_{X^{* *}}\left(f_{1}\right)=\left\{x^{* *} \in B_{X^{* *}}: x^{* *}\left(f_{1}\right)=\left\|f_{1}\right\|\right\}$. 
Now suppose, for an $i \in\{2, \ldots, n\}, J_{X}\left(f_{1}, \ldots, f_{i-1}\right)$ is defined and is a nonempty set. We define

$$
\begin{aligned}
M_{i} & =\sup \left\{f_{i}(x): x \in J_{X}\left(f_{1}, \ldots, f_{i-1}\right)\right\}, \\
M_{i}^{*} & =\sup \left\{x^{* *}\left(f_{i}\right): x^{* *} \in J_{X^{* *}}\left(f_{1}, \ldots, f_{i-1}\right)\right\}, \\
J_{X}\left(f_{1}, \ldots, f_{i}\right) & =\left\{x \in J_{X}\left(f_{1}, \ldots, f_{i-1}\right): f_{i}(x)=M_{i}\right\}, \\
J_{X^{* *}}\left(f_{1}, \ldots, f_{i}\right) & =\left\{x^{* *} \in J_{X^{* *}}\left(f_{1}, \ldots, f_{i-1}\right): x^{* *}\left(f_{i}\right)=M_{i}^{*}\right\} .
\end{aligned}
$$

For $\varepsilon>0$, let $J_{X}\left(f_{1}, \varepsilon\right)=\left\{x \in B_{X}: f_{1}(x)>\left\|f_{1}\right\|-\varepsilon\right\}$.

For $i=2, \ldots, n$, define

$$
J_{X}\left(f_{1}, \ldots, f_{i}, \varepsilon\right)=\left\{x \in J_{X}\left(f_{1}, \ldots, f_{i-1}, \varepsilon\right): f_{i}(x)>M_{i}-\varepsilon\right\} .
$$

Using a weak*-compactness argument, one can see that $J_{X^{* *}}\left(f_{1}, \ldots, f_{i}\right) \neq \varnothing$ for $i=1, \ldots, n$. In [6, Theorem 1], it is proved that if $Y$ is a finite co-dimensional proximinal subspace of $X$, then $J_{X}\left(f_{1}, \ldots, f_{i}\right) \neq \varnothing$ for $i=1, \ldots, n$ and for every basis $\left\{f_{1}, \ldots, f_{n}\right\}$ of $Y^{\perp}$.

For a finite co-dimensional subspace $Y$ of a Banach space $X$ and for a basis $\left\{f_{1}, \ldots, f_{n}\right\}$ of $Y^{\perp}$, we define the following sets as in [6].

$$
\begin{aligned}
\left(Y^{\perp}\right)^{*}\left(f_{1}\right) & =\left\{\phi \in\left(Y^{\perp}\right)_{1}^{*}: \phi\left(f_{1}\right)=\left\|f_{1}\right\|\right\}, \\
\left(Y^{\perp}\right)^{*}\left(f_{1}, \ldots, f_{i}\right) & =\left\{\phi \in\left(Y^{\perp}\right)^{*}\left(f_{1}, \ldots, f_{i-1}\right): \phi\left(f_{i}\right)=\max _{\psi \in\left(Y^{\perp}\right)^{*}\left(f_{1}, \ldots, f_{i-1}\right)} \psi\left(f_{i}\right)\right\} .
\end{aligned}
$$

We now obtain a relationship between the notions defined above.

Proposition 3.4. Let $Y \subset X$ be a subspace of finite co-dimension. Then $M_{i}^{*}=$ $\max \left\{\phi\left(f_{i}\right): \phi \in\left(Y^{\perp}\right)^{*}\left(f_{1}, \ldots, f_{i-1}\right)\right\}$ for $2 \leq i \leq n$ and for every basis $\left\{f_{1}, \ldots, f_{n}\right\}$ of $Y^{\perp}$.

Proof. Let $\left\{f_{1}, \ldots, f_{n}\right\}$ be a basis of $Y^{\perp}$. For $2 \leq i \leq n$, let

$$
\begin{aligned}
A_{i} & =\left\{\phi\left(f_{i}\right): \phi \in\left(Y^{\perp}\right)^{*}\left(f_{1}, \ldots, f_{i-1}\right)\right\}, \\
B_{i} & =\left\{\psi\left(f_{i}\right): \psi \in J_{X^{* *}}\left(f_{1}, \ldots, f_{i-1}\right)\right\} \text { and } \\
N_{i} & =\max \left\{\phi\left(f_{i}\right): \phi \in\left(Y^{\perp}\right)^{*}\left(f_{1}, \ldots, f_{i-1}\right)\right\} .
\end{aligned}
$$

Claim: $M_{2}^{*}=N_{2}$.

For, let $\phi \in\left(Y^{\perp}\right)^{*}\left(f_{1}\right)$. Now let $\tilde{\phi}$ be a Hahn-Banach extension of $\phi$ to $X^{*}$. Since $\|\tilde{\phi}\| \leq 1$ and $\tilde{\phi}\left(f_{1}\right)=\phi\left(f_{1}\right)=\left\|f_{1}\right\|, \tilde{\phi} \in J_{X^{* *}}\left(f_{1}\right)$. Then $\phi\left(f_{2}\right)=\tilde{\phi}\left(f_{2}\right) \in B_{2}$ and thus $A_{2} \subseteq B_{2}$. Therefore $N_{2} \leq M_{2}^{*}$.

On the other hand, let $\psi \in J_{X^{* *}}\left(f_{1}\right)$. Now let $\phi=\left.\psi\right|_{Y^{\perp}}$. Since $\|\phi\| \leq 1$ and $\phi\left(f_{1}\right)=\psi\left(f_{1}\right)=\left\|f_{1}\right\|, \phi \in\left(Y^{\perp}\right)^{*}\left(f_{1}\right)$. Thus $B_{2} \subseteq A_{2}$. Therefore $M_{2}^{*} \leq N_{2}$ and the claim follows.

Now let $i \in\{2, \ldots, n-1\}$ and let $M_{j}^{*}=N_{j}$ for $2 \leq j \leq i$. Let $\phi \in\left(Y^{\perp}\right)^{*}\left(f_{1}, \ldots\right.$, $\left.f_{i}\right)$. Now let $\tilde{\phi}$ be a Hahn-Banach extension of $\phi$ to $X^{*}$. Since $\tilde{\phi}\left(f_{j}\right)=\phi\left(f_{j}\right)=N_{j}=$ $M_{j}^{*}$ for $1 \leq j \leq i$, it follows that $\tilde{\phi} \in J_{X^{* *}}\left(f_{1}, \ldots, f_{i}\right)$. Then $A_{i+1} \subseteq B_{i+1}$ and hence $N_{i+1} \leq M_{i+1}^{*}$.

Let $\psi \in J_{X^{* *}}\left(f_{1}, \ldots, f_{i}\right)$. Now set $\phi=\left.\psi\right|_{Y^{\perp}}$. Since $\phi\left(f_{j}\right)=\psi\left(f_{j}\right)=M_{j}^{*}=N_{j}$ for $1 \leq j \leq i$, we have $\phi \in\left(Y^{\perp}\right)^{*}\left(f_{1}, \ldots, f_{i}\right)$. Then $B_{i+1} \subseteq A_{i+1}$ and hence $M_{i+1}^{*} \leq N_{i+1}$. Now the result follows by induction. 
By combining Proposition 3.4 and [6, Theorem 1], the following corollary is easy to see.

Corollary 3.5. Let $Y$ be a subspace of finite co-dimension in a Banach space $X$. Then $Y$ is proximinal in $X$ if and only if $J_{X}\left(f_{1}, \ldots, f_{i}\right) \neq \varnothing$ and $M_{i}=M_{i}^{*}$ for $1 \leq i \leq n$ and every basis $\left\{f_{1}, \ldots, f_{n}\right\}$ of $Y^{\perp}$.

The next set of results are needed to understand the implication of the strong proximinality of $Y^{\perp \perp}$ at points of $X$ in terms of $Y$. Some of the computations below are appropriate modifications of the arguments given in [4,7], we include these for the sake of completeness of the exposition.

Lemma 3.6. Let $Y$ be a subspace of a Banach space $X$. If $Y^{\perp \perp}$ is strongly proximinal at all $x \in X$, then for every $x \in X$ with $\|x\|=1=d(x, Y)$ and $\varepsilon>0$, there exists $a$ $\delta>0$ such that if $z \in B_{X}$ and $\|x-z+Y\|<\delta$, then there exists a $\phi \in B_{X^{* *} \text { such that }}$ $x-\phi \in Y^{\perp \perp}$ and $\|z-\phi\|<\varepsilon$.

Proof. Let $x \in X$ be such that $\|x\|=1=d(x, Y)$ and let $\varepsilon>0$. Since $Y^{\perp \perp}$ is strongly proximinal at $x$, there exists $0<\delta<\varepsilon / 2$ such that if $\varphi \in P_{Y \perp \perp}(x, \delta)$, then $d\left(\varphi, P_{Y \perp \perp}(x)\right)<\varepsilon / 2$. Hence for every $\varphi \in Q_{Y \perp \perp}(x, \delta)$, we have $d\left(\varphi, Q_{Y \perp \perp}(x)\right)<$ $\varepsilon / 2$.

Now let $z \in B_{X}$ and $\|x-z+Y\|<\delta$. Choose an element $y_{0}^{* *} \in P_{Y \perp \perp}(x-z)$ and let $w=x-z-y_{0}^{* *}$. Then $\|w\|<\delta$ and $f(w)=f(x-z)$ for all $f \in Y^{\perp}$. Now let $v=z+w$. Then $\|v\| \leq 1+\delta$ and $f(v)=f(x)$ for all $f \in Y^{\perp}$. Thus $v \in Q_{Y \perp \perp}(x, \delta)$. Then there exists a $\phi \in Q_{Y \perp \perp}(x)$ such that $\|v-\phi\|<\varepsilon / 2$. Since $\phi \in Q_{Y \perp \perp}(x), \phi \in B_{X^{* *}}$ and $x-\phi \in Y^{\perp \perp}$. Now the result follows from the fact that $\|z-\phi\| \leq\|z-v\|+\|v-\phi\|<\varepsilon$.

Lemma 3.7. Let $Y$ be a proximinal subspace of finite co-dimension in a Banach space $X$. If $Y^{\perp \perp}$ is strongly proximinal at $x$ for all $x \in X$, then for any basis $\left\{f_{1}, \ldots, f_{n}\right\}$ of $Y^{\perp}$,

$$
\lim _{\varepsilon \rightarrow 0} \sup \left\{d\left(x, J_{X^{* *}}\left(f_{1}, \ldots, f_{i}\right)\right): x \in J_{X}\left(f_{1}, \ldots, f_{i}, \varepsilon\right)\right\}=0 .
$$

Proof. Suppose $Y^{\perp \perp}$ is strongly proximinal at all $x \in X$ and let $\left\{f_{1}, \ldots, f_{n}\right\}$ be a basis of $Y^{\perp \perp \perp}=Y^{\perp}$. Let $\eta>0$ and $i \in\{1, \ldots, n\}$.

Now for $x \in X$ with $\|x\|=1=d(x, Y)$, there exists a $\delta_{x}>0$ such that

$$
\begin{aligned}
z \in B_{X},\|x-z+Y\|<\delta_{x} \Longrightarrow & \exists \phi \in B_{X^{* *}} \text { with } x-\phi \in Y^{\perp \perp} \text { and } \\
& \|z-\phi\|<\eta .
\end{aligned}
$$

If $\delta_{x}>0$ holds for an $x \in X$, then the same holds for all the elements in the coset $x+Y$. Also, if $x \in J_{X}\left(f_{1}, \ldots, f_{i}\right)$ then $\|x\|=1=d(x, Y)$. Now let $C_{i}=J_{X}\left(f_{1}, \ldots, f_{i}\right)$. Since $X / Y$ is finite dimensional, $C_{i}+Y$ is a compact subset of $B_{X / Y}$. Now for each element $x+Y \in C_{i}+Y$, let $\delta_{x}$ be as in (3.1). Then the open cover $\left\{B_{X / Y}\left(x+Y, \delta_{x}\right): x \in C_{i}\right\}$ of $C_{i}+Y$ has a finite subcover, say $\left\{B_{X / Y}\left(x_{j}+Y, \delta_{x_{j}}\right): 1 \leq j \leq m\right\}$.

Let $\delta=\min _{j} \frac{\delta_{x_{j}}}{2}$. We now claim that, since $Y$ is of finite co-dimension in $X$, there exists an $\varepsilon>0$ such that

$$
v \in J_{X}\left(f_{1}, \ldots, f_{i}, \varepsilon\right) \Longrightarrow d\left(v+Y, J_{X}\left(f_{1}, \ldots, f_{i}\right)+Y\right)<\frac{\delta}{2} .
$$


For, if not, then there exists a sequence $\left(v_{n}\right)$ in $J_{X}\left(f_{1}, \ldots, f_{i}, \frac{1}{n}\right)$ such that $d\left(v_{n}+Y, J_{X}\left(f_{1}, \ldots, f_{i}\right)+Y\right) \geq \frac{\delta}{2}$. Then by passing onto a subsequence, if necessary, we can assume that the sequence $\left(v_{n}+Y\right)$ converges, say to $v_{0}+Y$. Then $d\left(v_{0}+Y, J_{X}\left(f_{1}, \ldots, f_{i}\right)+Y\right) \geq \frac{\delta}{2}$. Now choose an element $y_{0} \in P_{Y}\left(v_{0}\right)$. Then $\left\|v_{0}-y_{0}\right\|=\left\|v_{0}+Y\right\| \leq 1$. Since $v_{n} \in J_{X}\left(f_{1}, \ldots, f_{i}, \frac{1}{n}\right)$ and $f_{j}\left(v_{n}\right)$ converges to $f_{j}\left(v_{0}\right)$ for $1 \leq j \leq i$, it follows that $f_{j}\left(v_{0}\right)=M_{j}$ for $1 \leq j \leq i$. Thus $v_{0}+y_{0} \in$ $J_{X}\left(f_{1}, \ldots, f_{i}\right)$, which is a contradiction since $d\left(v_{0}+Y, J_{X}\left(f_{1}, \ldots, f_{i}\right)+Y\right) \geq \frac{\delta}{2}$. Hence the claim follows.

Now let $v \in J_{X}\left(f_{1}, \ldots, f_{i}, \varepsilon\right)$. Then there exists an element $x_{0} \in J_{X}\left(f_{1}, \ldots, f_{i}\right)$ such that $\left\|v-x_{0}+Y\right\|<\frac{\delta}{2}$. Let $k \in\{1, \ldots, m\}$ be such that $\left\|x_{0}-x_{k}+Y\right\|<\frac{\delta_{x_{k}}}{2}$. Thus $\left\|v-x_{k}+Y\right\|<\delta_{x_{k}}$. Then there exists a $\phi \in B_{X^{* *}}$ such that $x_{k}-\phi \in Y^{\perp \perp}$ and $\|v-\phi\|<\eta$. Since $\phi\left(f_{j}\right)=f_{j}\left(x_{k}\right)=M_{j}=M_{j}^{*}$ for $1 \leq j \leq i$, we get $\phi \in J_{X^{* *}}\left(f_{1}, \ldots, f_{i}\right)$. Thus $d\left(v, J_{X^{* *}}\left(f_{1}, \ldots, f_{i}\right)\right)<\eta$ and hence the conclusion follows.

Now again using principle of local reflexivity one can prove that $d\left(x, J_{X^{* *}}\left(f_{1}, \ldots, f_{i}\right)\right)=d\left(x, J_{X}\left(f_{1}, \ldots, f_{i}\right)\right)$. We skip the proof of the following lemma as its proof is same as that of [7, Lemma 3.7] except for the following facts. The proof of [7, Lemma 3.7] requires the fact that $M_{i}=M_{i}^{*}$ which they obtained using the strong proximinality assumption on $Y$. But Corollary 3.5 shows that the proximinality assumption on $Y$ is enough to guarantee $M_{i}=M_{i}^{*}$.

Lemma 3.8. Let $Y$ be a proximinal subspace of finite co-dimension in a Banach space $X$ and let $\left\{f_{1}, \ldots, f_{n}\right\} \subseteq S_{Y^{\perp}}$ be any basis of $Y^{\perp}$. If $Y^{\perp \perp}$ is strongly proximinal at $x$ for all $x \in X$, then $d\left(x, J_{X^{* *}}\left(f_{1}, \ldots, f_{i}\right)\right)=d\left(x, J_{X}\left(f_{1}, \ldots, f_{i}\right)\right)$ for $1 \leq i \leq n$.

In [7], it is proved that if $Y$ is a finite co-dimensional proximinal subspace of $X$, then $Y$ is strongly proximinal in $X$ if and only if $Y^{\perp \perp}$ is strongly proximinal in $X^{* *}$. We now strengthen this result by requiring strong proximinality of $Y^{\perp \perp}$ only at elements of $X$.

Proposition 3.9. Let $Y$ be a proximinal subspace of finite co-dimension in a Banach space $X$. If $Y^{\perp \perp}$ is strongly proximinal at all points of $X$, then $Y^{\perp \perp}$ is strongly proximinal in $X^{* *}$.

Proof. Let $Y^{\perp \perp}$ be strongly proximinal at all points of $X$. Then for every basis $\left\{f_{1}, \ldots, f_{n}\right\}$ of $Y^{\perp}$, by Lemma 3.7 and Lemma 3.8, $\lim _{\varepsilon \rightarrow 0} \sup \left\{d\left(x, J_{X}\left(f_{1}, \ldots, f_{i}\right)\right)\right.$ : $\left.x \in J_{X}\left(f_{1}, \ldots, f_{i}, \varepsilon\right)\right\}=0$ for $1 \leq i \leq n$. Hence, by [4, Theorem 2.5], $Y$ is strongly proximinal in $X$. Then, by [7, Theorem 3.10], $Y^{\perp \perp}$ is strongly proximinal in $X^{* *}$.

The following theorem exhibits strong proximinality through a weak*-dense subset of $X^{* *}$.

Theorem 3.10. Let $X$ be a Banach space and $W$ be a finite co-dimensional weak ${ }^{*}$-closed subspace of $X^{* *}$ such that for every basis $\left\{f_{1}, \ldots, f_{n}\right\}$ of $W^{\perp}, J_{X}\left(f_{1}, \ldots, f_{i}\right) \neq \varnothing$ and $M_{i}=M_{i}^{*}$ for $1 \leq i \leq n$. If $W$ is strongly proximinal at all $x \in X$, then $W$ is strongly proximinal in $X^{* *}$. 
Proof. Since $W$ is a finite co-dimensional weak*-closed subspace of $X^{* *}$, there exists a basis $\left\{g_{1}, \ldots, g_{n}\right\} \subset X^{*}$ of $W^{\perp}$. Now let $Y=\bigcap_{i} \operatorname{ker}\left(g_{i}\right)$. Then $Y^{\perp \perp}=W$. Since $Y^{\perp}=Y^{\perp \perp \perp}=W^{\perp}$, each basis of $Y^{\perp}$ is also a basis of $W^{\perp}$. Thus for every basis $\left\{f_{1}, \ldots, f_{n}\right\}$ of $Y^{\perp}, J_{X}\left(f_{1}, \ldots, f_{i}\right) \neq \varnothing$ and $M_{i}=M_{i}^{*}$ for $1 \leq i \leq n$. Then, by Corollary 3.5, $Y$ is a proximinal in $X$. Thus, by Proposition 3.9, $W=Y^{\perp \perp}$ is strongly proximinal in $X^{* *}$.

We now give the proof of Theorem 3.3.

Proof of Theorem 3.3. If $Y^{\perp \perp}$ is strongly proximinal at all points of $C(K)$, then, by the proof of Proposition 3.9, $Y$ is strongly proximinal in $C(K)$. Hence, by Theorem 3.2 , the result follows.

\section{Acknowledgements}

A major part of this work was done when the first author was visiting Indian Statistical Institute, Bangalore and he would like to thank the Indian Statistical Institute for its financial support. The second author was a Fulbright-Nehru Academic and Professional Excellence Fellow at the Department of Mathematical Sciences, University of Memphis, when a part of this work was done.

\section{References}

[1] Ehrhard Behrends, On the principle of local reflexivity, Studia Math. 100 (1991), no. 2, 109-128.

[2] S. Dutta and Darapaneni Narayana, Strongly proximinal subspaces of finite codimension in $C(K)$, Colloq. Math. 109 (2007), no. 1, 119-128.

[3] A. L. Garkavi, The problem of Helly and best approximation in the space of continuous functions, Izv. Akad. Nauk SSSR Ser. Mat. 31 (1967), 641-656.

[4] G. Godefroy and V. Indumathi, Strong proximinality and polyhedral spaces, Rev. Mat. Complut. 14 (2001), no. 1, 105-125.

[5] G. Godini, Best approximation and intersections of balls, Banach space theory and its applications (Bucharest, 1981), 1983, pp. 44-54.

[6] V. Indumathi, Proximinal subspaces of finite codimension in general normed linear spaces, Proc. London Math. Soc. (3) 45 (1982), no. 3, 435-455.

[7] C. R. Jayanarayanan and T. Paul, Strong proximinality and intersection properties of balls in Banach spaces, J. Math. Anal. Appl. 426 (2015), no. 2, 12171231.

[8] W. A. Light, Proximinality in $L_{p}(S, Y)$, Rocky Mountain J. Math. 19 (1989), no. 1, 251-259.

[9] W. A. Light and E. W. Cheney, Approximation theory in tensor product spaces, Lecture Notes in Mathematics, vol. 1169, Springer-Verlag, Berlin, 1985. 
[10] José Mendoza, Proximinality in $L_{p}(\mu, X)$, J. Approx. Theory 93 (1998), no. 2, 331-343.

[11] Ivan Singer, Best approximation in normed linear spaces by elements of linear subspaces, Die Grundlehren der mathematischen Wissenschaften, Band 171, Publishing House of the Academy of the Socialist Republic of Romania, Bucharest, 1970.

[12] S. M. Srivastava, A course on Borel sets, Graduate Texts in Mathematics, vol. 180, Springer-Verlag, New York, 1998.

[13] David Yost, The n-ball properties in real and complex Banach spaces, Math. Scand. 50 (1982), no. 1, 100-110.

Department of Mathematics and Statistics, Indian Institute of Technology, Kanpur 208016, India

E-mail : crjayan@iitk.ac.in

Theoretical Statistics and Mathematics Division, Indian Statistical Institute, R. V. College Post, Bangalore 560059, India

E-mail : tss@isibang.ac.in

srin@fulbrightmail.org 